\title{
Coherent Ultra-Dense WDM-PON Enabled by Complexity-Reduced Digital Transceivers
}

\author{
Jeison Tabares, Saeed Ghasemi, J. Camilo Velásquez, and Josep Prat, Member, IEEE
}

\begin{abstract}
Coherent technologies along with digital signal processing (DSP) have revolutionized the optical communication systems, significantly increasing the capacity of the fiber channel owing to transmission of advanced modulation formats and mitigation of propagation impairments. However, the commercial solutions for high-capacity core networks are too complex and costly, then hardly feasible, for access networks with high terminal density, where cost, power budget and footprint are the main limiting factors. This article analyses the key enabling techniques to implement a complexity-reduced coherent transceiver (CoTRX) by exploiting photonic integration, simplified optical modulation, low-cost DFB lasers, consumer electronics and low-complexity DSP. Bulk optical modulators are replaced by direct amplitudeand-phase modulation of integrated electro-absorption modulated laser (EML) with smaller footprint, generating up to 8-ary modulation formats. Hardware-efficient DSP algorithms for the coherent transmitter and receiver, including pulse-shaping for direct phase modulation, differential detection for optical phase recovery, and digital pre-emphasis with enhanced tolerance to quantization noise, are investigated to face the challenges imposed by low-cost photonic and electronic devices, such as strong phase noise, wavelength drifts, severe bandwidth limitation, and low resolution data converters. Through numerical simulations and real-time experiments, the results indicate that this new class of CoTRX enables effective implementation of wavelength-to-theuser PON with dedicated 1.25 to $20 \mathrm{~Gb} / \mathrm{s}$ per user, in an ultradense 6.25 to $25 \mathrm{GHz}$ spaced WDM optical grid, with $>30 \mathrm{~dB}$ loss budget, outperforming the current competing technologies for access networks.
\end{abstract}

Index Terms - Coherent detection, electro-absorption modulated laser, wavelength division multiplexing, digital signal processing, access networks.

\section{INTRODUCTION}

$\mathbf{T}$ he standardized and commercially deployed optical access networks need to evolve facing the upcoming bandwidthhungry multimedia services with ultra-high definition video, business connectivity and mobile front-haul/back-haul (MFH/MBH) for 5G. The current passive optical networks (PONs) attending the XG-PON standard rely on $10 \mathrm{~Gb} / \mathrm{s}$ data rate, serving the final subscribers by time division multiplexing (TDM). To enhance the total PON capacity, the latest ITU-T standard NG-PON2 exploits the wavelength division multiplexing (WDM) by stacking 4/8 XG-PON wavelengths

Manuscript submitted June 28, 2019. This work was supported in part by the Spanish Project FLIPER (TEC2015-70835), and the FPU program from the Education Ministry of Spain (FPU12/06318).

The authors are with the Universitat Politècnica de Catalunya, Signal Theory and Communications Department (TSC), Barcelona E-08034, Spain (email:

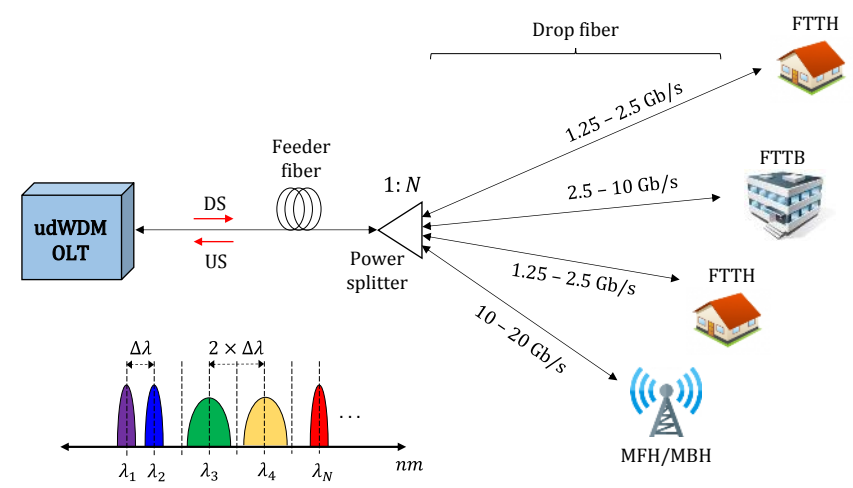

Fig. 1. Flexible coherent udWDM-PON with dedicated $\lambda$ per user supporting different types of services. DS: downstream, US: upstream, FTTH: fiber to the home, FTTB: fiber to the business.

( $\lambda$ ) achieving aggregate PON capacity up to $80 \mathrm{~Gb} / \mathrm{s}$ [1]. However, scaling the actual time-and-wavelength division multiplexing (TWDM) technology to higher data rates in a costeffective way might not be compatible with low-cost commercial photonic and electronic devices, mandatory for affordable PON implementation. Specifically, increasing the bit rate per single $\lambda$ constitutes a high cost and energy consuming solution due to the large required bandwidth (BW) of the hardware (HW) devices such as lasers/modulators, photodiodes (PDs), and amplifiers. Moreover, transmission impairments like chromatic dispersion (CD) become more apparent on large BW signals, requiring digital signal processing (DSP) for mitigation with necessary data converters that are expensive and power-hungry if the required $\mathrm{BW}$ and sampling rate are high.

The novel solutions for next generation PONs must be: (I) affordable in cost by the final users, (II) fully compatible with legacy access systems based on power splitting at the outside plant, and (III) flexible in terms of user BW and channel allocation, with reconfigurable transceivers (TRXs) enabling convergence of residential, business and MFH/MBH services. A key technology fulfilling these requirements is a new class of coherent TRX (CoTRX) implementing the $\lambda$-to-the-user concept, where each subscriber owns a dedicated $\lambda$ in a costeffective manner by multiplexing in ultra-dense WDM (udWDM). The bit rate per $\lambda$ retains compatible with consumer

jtabares@tsc.upc.edu; saeed.ghasemi@tsc.upc.edu; juavelasquez@tsc.upc.edu; jprat@tsc.upc.edu). 

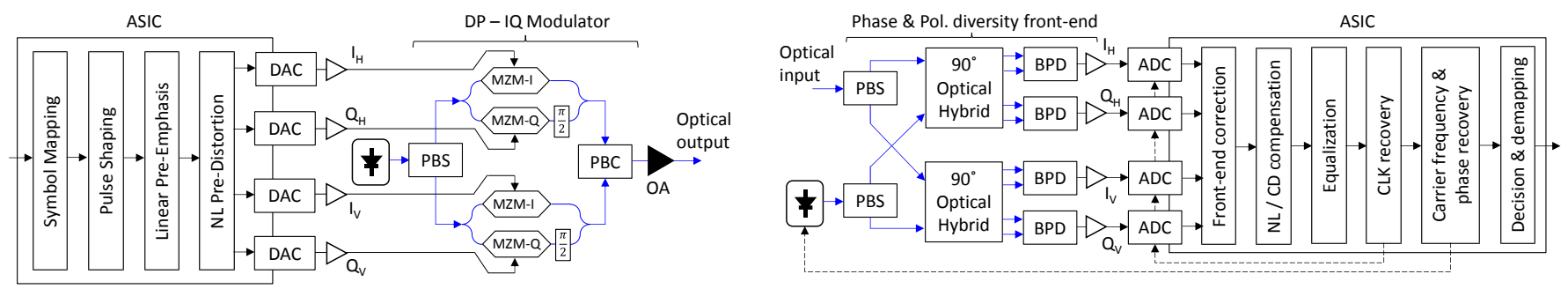

Fig. 2. Block diagram of a conventional CoTRX for high-capacity optical networks: TX (left) and RX (right). OA: optical amplifier; MZM: Mach-Zehnder modulator; PBS/PBC: polarization beam splitter/combiner.
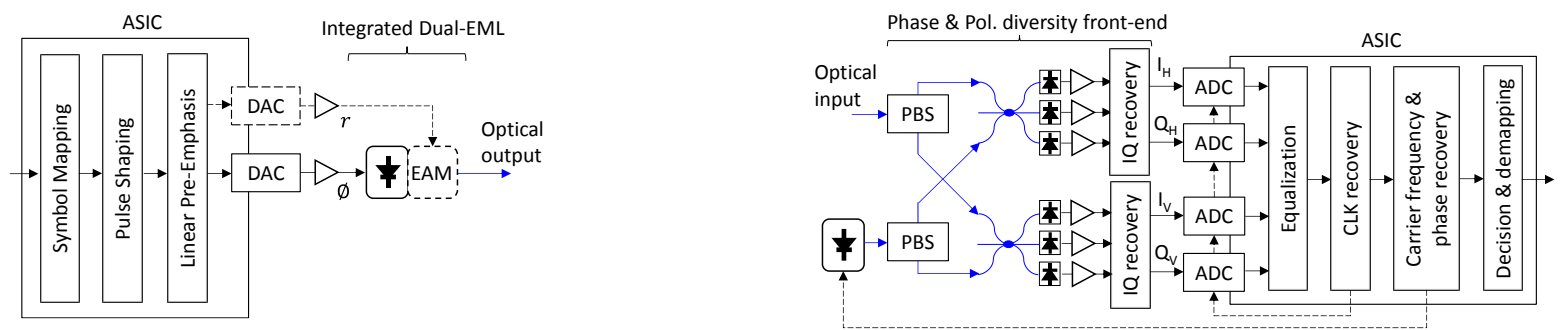

Fig. 3. Block diagram of the proposed CoTRX for udWDM-PON with simplified architecture: TX (left) and RX (right).

electronics BW, but still achieving superior aggregate PON capacity with high spectral efficiency (SE) [2], [3] compared with standard access systems. The CoTRX technology includes commercial low-cost distributed feed-back (DFB) or verticalcavity surface-emitting laser (VCSEL) as optical sources, flexible optical grid with limited $\lambda$ tunability, low-speed data converters, and low-complexity DSP. The DSP plays the fundamental role of enhancing both the system capacity and the SE by transmitting advanced modulation formats, as well as compensating for all the distortions and transmission impairments that make impact on the signal from origin to destination.

Fig. 1 depicts the flexible udWDM-PON enabled by coherent technologies, supporting different applications over the same fiber infrastructure deployed for current PONs. The optical distribution network (ODN) is totally passive and filterless, with user terminals implementing tunable lasers with nonpreselected $\lambda$. Channel selection is done by $\lambda$-tuning of the local oscillator (LO) laser at the CoTRX, allowing for ultra-narrow spaced WDM grid compared with conventional selectivity by optical filters. The link power budget is a critical factor to be addressed in next generation PONs [1], [4]; owing to the superior sensitivity of coherent detection, the udWDM-PON can achieve the targeted power budget $>30 \mathrm{~dB}$ without increasing the launched power or extra optical amplification, thus lowering the total energy consumption.

In this article, we discuss how the optical coherent technologies supported by DSP, that are mature in highcapacity and long-haul transport networks, can be migrated for access applications or, in general, optical networks with high terminal density. We explore photonic integration of low-cost DFBs with electro-absorption modulator (EAM), along with direct intensity-and-phase modulation leveraging the laser chirp, as a key technology to reduce the complexity and energy consumption of the coherent transmitter (TX). With this small footprint device, up to 8-amplitude-and-phase shift keying (8APSK) at $7.5 \mathrm{~Gb} / \mathrm{s}$ was achieved with RX sensitivity of -40 $\mathrm{dBm}$. Besides, this article analyzes HW-efficient DSP algorithms to transmit advanced modulation formats, dealing with the impairments from low-cost photonic/electronic devices. Low-resolution and band-limited data converters have been considered, adapting the pre-emphasis DSP algorithm of the TX to operate in presence of quantization noise. Simple digital pulse-shaping at the TX DSP is proposed to generate complex phase-modulated signals exploiting the laser chirp. Straightforward differential detection is investigated for carrier recovery $(\mathrm{CR})$ at the receiver $(\mathrm{RX})$, showing high tolerance to strong phase noise, up to total spectral linewidth $\Delta v=0.64 \%$ of the bit rate for differential PSK (DPSK) in real-time.

The outcome is a complexity-reduced and cost-effective digital CoTRX for udWDM-PON that is conceptually complex, but affordable in cost by exploiting photonic integration, simplified optical modulation, low-cost consumer electronics and HW efficient DSP. Despite the CoTRXs simplifications, the udWDM-PON outperforms the present PON technologies achieving loss budget in excess of $30 \mathrm{~dB}$, enabling a large number of users in a 6.25 to $25 \mathrm{GHz}$ spaced optical grid for 1.25 to $20 \mathrm{~Gb} / \mathrm{s} \lambda$-to-the-user access network, totally passive, transparent, and fully compatible with deployed legacy PON systems.

The article is organized as follows. Section II presents the architecture of the simplified CoTRX for udWDM-PON, inherited from conventional CoTRX technology along with novel strategies reducing the complexity and cost. The DSP algorithms for the TX and RX subsystems are analyzed in Sections III and IV respectively, followed by the conclusions in Section V.

\section{Coherent Transceivers Technology}

The actual commercially available CoTRXs are mainly 
intended for high capacity core networks supporting large volumes of data traffic. They are also gaining interest for shortreach data center interconnect (DCI) to increase the total data rate within the same BW. Performance evaluation of CoTRXs for 400G DCI systems reveals that the overall complexity and energy consumption is comparable to that the intensitymodulation direct-detection (IM-DD) counterpart, that dominates the DCI market segment, but with superior power budget and SE [5].

To correctly transport such high data rates with CoTRXs, information is mapped into advanced modulation formats with dense complex constellations that propagate through electronic and optical devices of the CoTRX, and through the transmission media, i.e., the optical fiber infrastructure.

Fig. 2 depicts a block diagram of the conventional CoTRX with all the HW components and the DSP subsystems [6]. In general, the CoTRXs are still complex and too expensive for optical access with high terminal density. Moreover, the necessary digital-to-analog/analog-to-digital converters (DACs/ADCs) are usually listed as critical elements since their cost and power consumption increases if the required sample rate, resolution bits, and $\mathrm{BW}$ are high. However, during the last years efforts in research are being taken to develop CoTRXs with simplified architectures and HW efficient DSP [7]-[11]. Yet, most of the proposed solutions still rely on external modulators, or extra RX BW that unfavorably scales with the bit rate. A recent alternative for CoTRXs is the Kramers-Kronig $\mathrm{RX}$ [12] that substantially reduces the RX front-end to a single PD per each state of polarization (SOP), but does not exhibit the high sensitivity and $\lambda$-selectivity of coherent systems. Also, the required $\mathrm{BW}$ twofold compared with coherent homodyne detection.

In practice, the adoption of digital coherent systems for PONs will depend on (I) reducing the footprint of the CoTRX photonic elements; (II) lowering the requirements for ADCs and DACs in terms of BW, resolution, and sample rate; (III) simplifying the DSP complexity, thus reducing the energy consumption and overall cost of the digital processor.

Fig. 3 presents the proposed realization of a CoTRX for udWDM-PON addressing the aforementioned requirements. First, for generation of advanced modulation formats the inphase and quadrature (IQ) modulators represent a severe limitation in terms of footprint, cost, and power budget. Instead, we successfully proposed and demonstrated in [13] a simple $m$ ary coherent TX based on dual electro-absorption modulated laser (Dual-EML) for up to 8-APSK.

The Dual-EML is a monolithically integrated photonic device where the same active layer is composed by two sections, a DFB and an EAM. The term dual denotes that the EML chip has dual electrodes with independent radio frequency (RF) inputs to simultaneously phase-modulate the DFB and intensity-modulate the EAM. Careful optimization of the bias current/voltage is necessary to isolate the two modulations generated by the Dual-EML. First, the DFB section is biased far from the threshold current, minimizing the extinction ratio of the residual IM at most, thus behaving the DFB as a pure optical frequency modulator (FM) by the laser chirp. Similarly, the FM contribution of the EAM by its inherent chirp can be reduced by biasing the EAM near to the zero-chirp region, as reported in Fig. 6 of [13]. Optical amplification is not necessary since no external power-losing devices are attached to the Dual-EML output, lowering the power consumption of the coherent TX.

At the RX front-end, the $90^{\circ}$ optical hybrids in Fig. 2 are replaced by much simpler $3 \times 3$ fiber couplers in Fig. 3 . This allows for phase-and-polarization diversity with six singleended PDs, two less than the four balanced PDs (BPD). The IQ signals for each SOP are recovered by linearly combining the three photocurrents in passive HW [14]. Note that the IQ recovery could be implemented into the DSP; however, this simple analog pre-processing saves two ADC channels that are critical components of the RX.

To further reduce the complexity and cost, the CoTRX should resort on the use of commercial low-cost lasers, such as DFBs with statistical (i.e. non-preselected) $\lambda$. This in turn, imposes extra challenges as the DFBs exhibit $\lambda$-instabilities, high $\Delta v$, and limited tunability. The DSP algorithms of the CoTRX mitigate the first two impairments $(\lambda$-drifts, $\Delta v)$, that directly affect data making strong impact on the received constellation, as it will be treated in Section IV.

On the other hand, the limited thermal tunability of the DFBs is critical for the control of the TX and LO lasers in the udWDM-PON, featuring hundreds of $\lambda \mathrm{s}$ in a narrow-spaced optical grid. Typical DFBs have a tunability ratio of $\sim 0.1 \mathrm{~nm} /$ ${ }^{\circ} \mathrm{C}$ [15] covering $\sim 3 \mathrm{~nm}(375 \mathrm{GHz})$ by $\pm 15{ }^{\circ} \mathrm{C}$ temperature range, large enough to spread over many, though not all, udWDM channels. To properly manage the $\lambda$ activation process and operation, a set of heuristic channel assignment algorithms with dynamic wavelength allocation (DWA) have been proposed to avoid collisions and efficiently organize the udWDM spectrum, lowering the blocking probability of new users connecting to the PON and satisfying network changes in environmental temperature [16].

The DSP of the CoTRX can be implemented either in an application-specific integrated circuit (ASIC) or in a fieldprogrammable gate array (FPGA). The digital CoTRX reconfigures easily dynamically adjusting its parameters such as modulation format and data BW, adapting to the type of service and user. This is advantageous for network flexibility compared with analog systems that require HW changes.

Some of the DSP algorithms of the conventional CoTRX in Fig. 2 are key functionalities to correctly generate and detect the modulated data, while others related to equalization and impairments mitigation play a secondary role in the access scenario with lower data rates, simpler modulation formats, and shorter fiber spans. Next sections analyze all the proposed DSP algorithms of the CoTRX in Fig. 3, intended for cost-effective udWDM-PON.

\section{DSP SUBSYSTEMS OF THE TRANSMITTER}

In the simplified TX of Fig. 3, the bulk dual-polarization $I Q$ (DP-IQ) modulator is replaced by the EAM integrated into the Dual-EML. The EAM has a non-linear (NL) electro-optic response, similar to Fig. 3 of [13]. Nevertheless, the EAM bias 


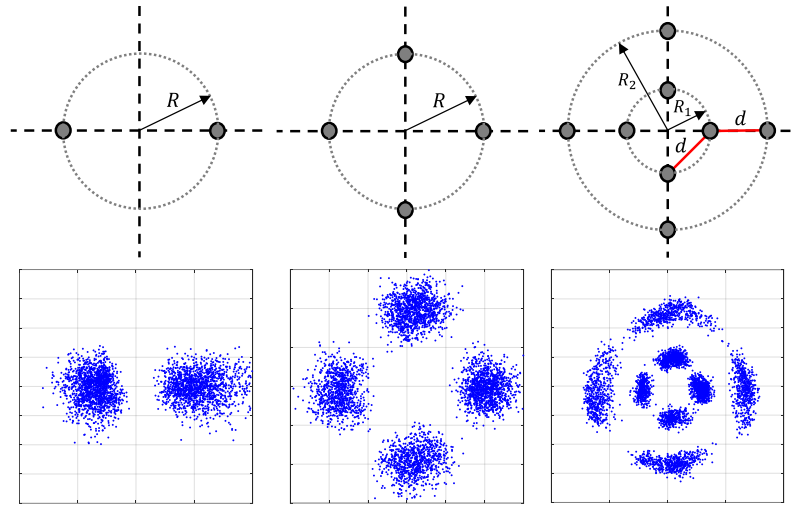

Fig. 4. BPSK, QPSK, and 8-APSK experimental constellation diagrams for direct modulation with the Dual-EML.

and the extinction ratio (ER) can be adjusted to operate in the linear region, and the NL pre-distortion by DSP might be optional or discarded.

Therefore, the key DSP subsystems for the TX in Fig. 3 are: symbol mapping, pulse shaping and linear pre-emphasis.

\section{A. Symbol Mapping}

In the conventional CoTRX of Fig. 2, the DP-IQ modulator creates a quadrature amplitude modulation (QAM) with rectangular constellation in the complex plane by each pair of coordinates $I, Q$. In contrast, the Dual-EML in Fig. 3, proposed as a cost-effective complex optical modulator, generates a circular constellation with polar coordinates $r, \phi$ by directly modulating the intensity with the EAM and the phase with the DFB laser chirp. In terms of flexibility, the TX is able to generate a set of circular modulation formats spanning from binary PSK (BPSK) up to at least 8-APSK, without adding extra HW complexity. Fig. 4 shows the constellation diagram for BPSK, quadrature PSK (QPSK) and 8-APSK, as well as the experimental constellations obtained from direct amplitude-and phase-modulation of the Dual-EML at $2.5 \mathrm{GBd}$. It is worth noting the phase-widening of the constellation points for 8APSK, compared to that of BPSK and QPSK where the EAM is off, due to the increment of the spectral linewidth along with the residual chirp of the EAM under modulation.

For phase modulation only, the constellation symbols are $s_{k}=R e^{j \phi_{k}}$, with $R$ the constant radius and $\phi_{k}$ the phaseencoded data defined by $\phi_{k}=\{0, \pi\}$ and $\phi_{k}=\left\{0, \pm \frac{\pi}{2}, \pi\right\}$ for BPSK and QPSK respectively. The mean symbol power is given by $\bar{\Gamma}=R^{2}$. In the case of amplitude and phase modulation, consecutive symbols are set to have symmetric Euclidean distance $d$ among them. Hence, for 8-APSK the symbols are defined by $s_{k}=r_{k} e^{j \phi_{k}}$, with $r_{k}=\left\{R_{1}, R_{2}\right\}$ and $\phi_{k}=\left\{0, \pm \frac{\pi}{2}, \pi\right\}$ the amplitude and phase information respectively. The ratio between radii for symmetric $d$ is found to be $R_{2} / R_{1}=1+\sqrt{2}$.

Table I summarizes our reported experiments with direct amplitude-and-phase modulation of low-cost DFBs and DualEML, with different modulation formats. Results show good performance with high RX sensitivity, measured for a bit error ratio (BER) of $10^{-3}$, fully compatible with current forward error correction (FEC) systems. Notably, 8-APSK shows $8.5 \mathrm{~dB}$ better sensitivity than 8-PSK, at the same bit rate and comparable $\Delta v$, due to less impact of the phase noise on the underlying QPSK of the 8-APSK. Considering the worst Rx sensitivity ( $-31.5 \mathrm{dBm}$ for 8 -PSK at $7.5 \mathrm{~Gb} / \mathrm{s})$, with a launched power of $0 \mathrm{dBm}$ per user, a power loss budget $>30 \mathrm{~dB}$ is achieved fulfilling the requirements of next generation access [1]. In terms of channel spacing, we highlight that the 8-(A)PSK formats, that provide the highest bit rate $(7.5 \mathrm{~Gb} / \mathrm{s})$, can be fitted in $6.25 \mathrm{GHz}$ spaced udWDM channels, enhancing the SE and throughput of the PON.

TABLE I

Performance of Direct Amplitude-AND-Phase Modulation

\begin{tabular}{lccr}
\hline \multicolumn{1}{c}{ Format } & Bit rate $[\mathrm{Gb} / \mathrm{s}]$ & Sensitivity $[\mathrm{dBm}]$ & \multicolumn{1}{c}{ Remarks } \\
\hline BPSK & 1.25 & -55 & Real-time [14] \\
QPSK & 5 & -44 & {$[13]$} \\
8-PSK & 7.5 & -31.5 & {$[17]$} \\
4-APSK & 5 & -40 & Heterodyne [18] \\
8-APSK & 7.5 & -40 & {$[13]$} \\
\hline
\end{tabular}

This technique can be directly extended for higher order modulation formats with more amplitude and phase levels, depending on the total laser $\Delta v$ and the signal-to-noise ratio (SNR) constraints. Moreover, if only phase modulation is generated, the TX in Fig. 3 only requires a laser and one DAC channel further reducing the complexity, cost and energy consumption.

\section{B. Pulse Shaping}

The pulse shaping is a key enabling technology to simplify the complexity of the optical TX, as depicted in Fig 3. It performs two main functionalities: (I) the proper adaptation of the modulating current for direct phase modulation with the laser chirp, and (II) the conventional spectral shaping for suppression of the out-band modulated signal power.

Regarding the first, it exploits the fact that when a semiconductor laser is under direct current modulation, the emission optical frequency $v(t)$ varies according to variations in the optical power $P(t)$, due to the chirp parameter of the laser. This phenomena was early described in [19], [20], giving the expression for the optical frequency variation

$$
\Delta v(t)=\frac{\alpha}{4 \pi}\left(\kappa P(t)+\frac{1}{P(t)} \frac{d P(t)}{d t}\right)
$$

where $\alpha$ is the linewidth enhancement factor or Henry coefficient, and $\kappa$ is the adiabatic chirp coefficient. The first term in Eq. (1) is the adiabatic chirp, whereas the second term is the transient chirp.

For direct phase modulation, the laser is biased at large values while the current swing for modulation is usually small, producing variations of the optical power that are significantly lower than the mean emitted power. In addition, the adiabatic chirp is dominant at mid frequencies (up to several $\mathrm{GHz}$ ) whereas the transient chirp is more apparent at high frequencies during fast symbol transitions.

Under this conditions, and considering the data BWs for 


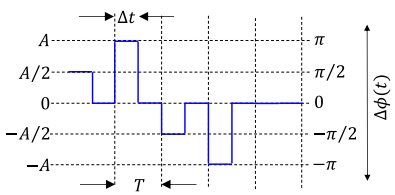

(a)

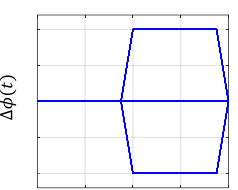

(b)

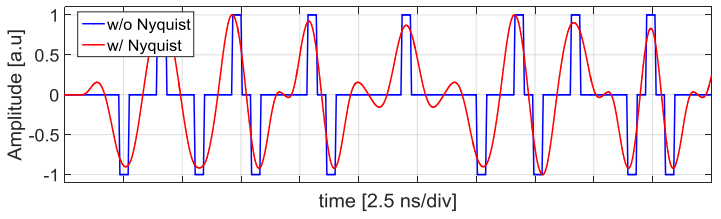

(c)

Fig. 5. (a) $50 \%$ D-C RZ waveform for direct QPSK modulation; (b) 50\% D-C RZ eye diagram for direct BPSK modulation with (right) and without (left) Nyquist filtering (roll-off $=0.25$ ); (c) time domain waveforms of (b).

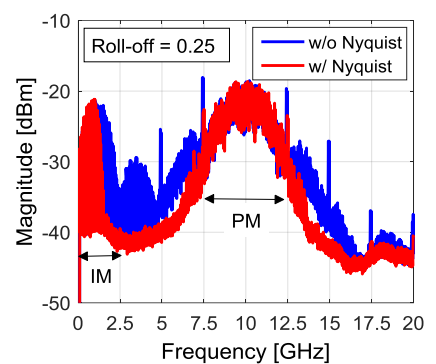

(a)

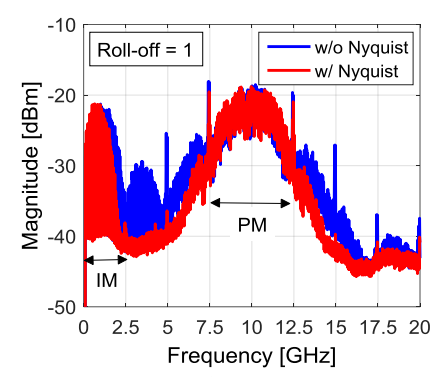

(b)
Fig. 6. Heterodyning photodetected spectra for BPSK at $2.5 \mathrm{~Gb} / \mathrm{s}$ and Nyquist filtering with (a) 0.25 and (b) 1 filter roll-off.

PON, the adiabatic chirp in Eq. (1) prevails and the semiconductor laser can be modelled as an optical FM modulator with residual IM, corresponding to the small-signal approach to the laser rate equations [21]. Hence, for direct phase modulation lasers with large $\kappa$ are optimal to lower the residual IM. Experimental characterization of a commercial DFB biased at $80 \mathrm{~mA}$, with modulation current swing of $11 \mathrm{~mA}$, resulted in $\kappa=10.8 \mathrm{GHz} / \mathrm{mW}$ with residual IM of about $1 \mathrm{~dB}$, for direct BPSK at $1.25 \mathrm{~Gb} / \mathrm{s}$. The average emitted power of the DFB was $2 \mathrm{dBm}$.

To translate the chirp FM modulation into phase modulation (PM), the desired phase shift $\Delta \phi(t)$ is achieved by controlling both the frequency deviation $\Delta v(t)$ and the time duration $\Delta t$ of the modulating pulse, according to the expression $\Delta \phi(t)=$ $2 \pi \Delta v(t) \Delta t$. As an example, for a reference phase shift of $\pi$ the product $\Delta v(t) \Delta t$ equals to 0.5 .

The result is a return-to-zero (RZ) pulse shape with variable duty-cycle (D-C) that drives the direct phase modulation of the laser [22], as represented in Fig. 5a for QPSK. Note that for data symbols with zero phase-shift, no current swing is applied to the laser during the symbol time $T$, producing zero-crossing in the eye diagram of the driving signal, as appreciated in Fig. 5b for direct BPSK modulation.

Furthermore, Nyquist filtering can be applied to reduce the modulated total spectral width and suppress the unwanted spectral side-lobes that may cause severe interference to adjacent channels in the udWDM-PON, with narrow channel spacing. The eye diagram and time-domain waveform of the direct-BPSK modulating current are plotted in Fig. 5b and 5c respectively, for $50 \%$ D-C RZ with and without Nyquist filtering (roll-off $=0.25$ ).

An important remark is that the Nyquist filtering eliminates the modulation side-lobes, but the filter roll-off has no impact on the main lobe BW, because of the direct phase modulation that relies on NL exponential modulation of the laser chirp, with strong harmonic dynamics [21], [23]. This phenomena can be appreciated in Fig. 6, that plots the experimental photodetected spectra for BPSK at $2.5 \mathrm{~Gb} / \mathrm{s}$, centered at $10 \mathrm{GHz}$, for two rolloff values of the Nyquist filter. The width of the PM modulation main lobe retains unchanged regardless the filter roll-off, while the width of the residual IM in the base-band is narrowed since the IM is generated by a linear modulation.

In a real PON scenario with direct phase modulation, the benefit of Nyquist spectral shaping becomes apparent when maximum $15 \mathrm{~dB}$ differential optical path losses are allowed, as specified in [1] for NG-PON2, due to the strong interference of modulation side lobes on weaker adjacent channels. The results reported in [23] indicate $25 \%$ reduction in channel spacing for direct phase modulation with Nyquist shaping, allowing that 2.5 $\mathrm{Gb} / \mathrm{s}$ BPSK users having $15 \mathrm{~dB}$ power difference between adjacent channels can be fitted in a $6.25 \mathrm{GHz}$ spaced optical grid.

\section{Linear Pre-Emphasis}

The simplified optical TX for PONs, that employs direct modulation of low-cost DFB, VCSEL or Dual-EML, might present severe BW limitation and non-flat frequency response. Furthermore, to keep the overall cost of the CoTRX down, the low-cost data converters (DACs and ADCs) might also present narrow BW and low-resolution, quantified through the effective number of bits (ENOB).

The linear digital pre-emphasis (DPE) at the TX DSP jointly mitigates the $\mathrm{BW}$ limitation and flattens the frequency response of the laser, driver amplifier, and DAC. For this purpose, the well-known zero-forcing (ZF) equalizer $P(f)=H^{-1}(f)$, being $H(f)$ the frequency response of the TX HW, could cancel all linear distortion and inter-symbol interference (ISI). However, it presents some disadvantages since it might over-amplify the noise greatly in the spectral region where $H(f)$ is more attenuated. It might also exhibit large peaks in the time domain signal that could lead to driver amplifier saturation and clipping. Instead, a linear DPE calculated trough the minimum mean squared error (MMSE) between the desired and the real system output, does not eliminate the interference completely but minimizes the total power of the noise and the interference components [24]. Precisely, it is optimal in presence of significant quantization noise due to DACs/ADCs with low ENOB, as exhibited by low-cost data converters suitable for PONs.

The DPE calculated through the MMSE is designed in the frequency domain as a linear filter $P(f)$ following the analytical derivation in [25], [26], with respect to the TX in Fig. 3 , leading to 


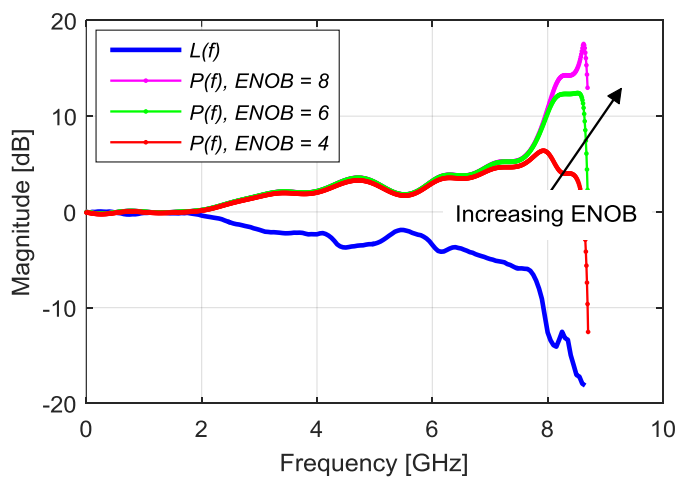

Fig. 7. DPE frequency response when varying the ENOB of the DAC.

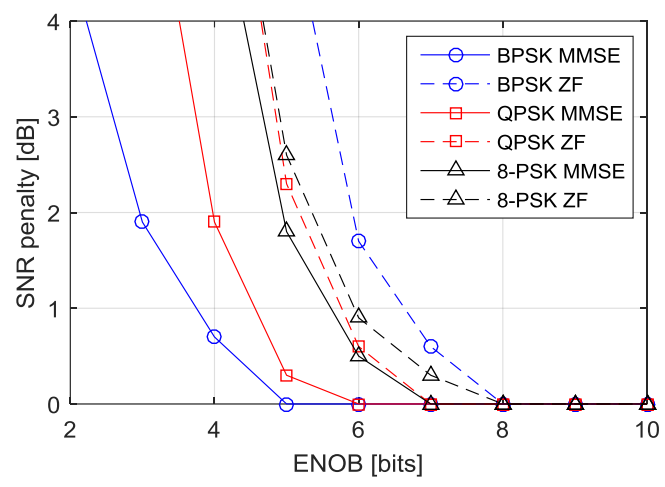

Fig. 8. SNR penalty at BER $=10^{-4}$ vs. ENOB, for ZF-DPE and MMSE-DPE comparison with different modulation formats.

$$
\operatorname{MMSE}(P(f))=\min _{P(f)}^{R_{B}} \int_{-R_{B}}^{R_{B}}\left[\left|y(f)-y_{i d}(f)\right|^{2}\right] d f
$$

where $\mathrm{E}[\cdot]$ is the expectation operator, $R_{B}$ is the symbol rate, $y_{i d}(f)$ is the ideal system output, and $y(f)$ is the real system output affected by the laser $L(f)$ and DAC $D(f)$ frequency responses respectively. After solving for Eq. (2), the analytical expression of the DPE filter $P(f)$ reads as

$$
P(f)=\frac{D^{*}(f) L^{*}(f)}{|D(f) L(f)|^{2}+\xi_{0}}
$$

The parameter $\xi_{0}$ takes into account the peak-to-average power ratio (PAPR) of the signal, and the quantization noise variance of the DAC through the ENOB. The $\xi_{0}$ is calculated as

$$
\xi_{0}=\frac{10^{\frac{P A P R_{d B}}{10}}}{6 R_{B} \cdot 2^{2 E N O B}} \int_{-R_{B}}^{R_{B}}|D(f) L(f)|^{2} d f
$$

Fig. 7 reports the experimental characterization of the FM modulation response $L(f)$ of a common DFB, showing BW limitation with $\mathrm{BW}_{3 \mathrm{~dB}}=\sim 5 \mathrm{GHz}$, and non-flat response with about $2.5 \mathrm{~dB}$ ripple in the pass-band. In addition, the ENOB influence on the DPE filter response $P(f)$ is also clearly visible in Fig. 7. The ENOB, included in the $\xi_{0}$ calculation, determines the optimal roll-off of $P(f)$ minimizing over-amplification of the quantization noise. Note that for high ENOB, the $\xi_{0}$ reduces and the DPE filter in Eq. (3) converges to the ZF equalizer $P(f)=D^{-1}(f) L^{-1}(f)$, which is sub-optimal if the quantization noise dominates at the TX.

In our previous work [25], numerical simulation was carried out by employing $L(f)$ of Fig. 7 to determine the maximum transmission rate using a commercial DFB with direct phase modulation and DPE at the TX. Direct BPSK, QPSK and 8PSK were generated, achieving $R_{B}=\{11,9.5,7.5\}$ GBd respectively for $1 \mathrm{~dB}$ SNR penalty at $\mathrm{BER}=10^{-4}$, with respect to an ideal laser response. It translates into $\{11,19,22.5\} \mathrm{Gb} / \mathrm{s}$ enabled by 32-tap DPE linear filtering. The DAC BW was 8 $\mathrm{GHz}$, and the ENOB was modelled as variable.

Here, we extend the analysis to assess the impact of the quantization noise from the DAC on the DPE calculated through the MMSE and the ZF. The results are shown in Fig. 8, in terms of the SNR penalty at BER $=10^{-4}$ as a function of the DAC ENOB. As observed, the MMSE better tolerates the quantization noise than the $\mathrm{ZF}$ for all modulation formats, with similar implementation complexity of the DPE filter. In our simulation, employing $L(f)$ of Fig. 7 with $\mathrm{BW}_{3 \mathrm{~dB}}=\sim 5 \mathrm{GHz}$, BPSK reached the highest $R_{B}(11 \mathrm{GBd})$ for $1 \mathrm{~dB}$ SNR penalty thus it is the most affected by low ENOB for the case of ZF compared with MMSE, because the ZF further enhances the quantization noise at the high frequencies beyond $5 \mathrm{GHz}$. Accordingly, 8-PSK achieved the lowest $R_{B}(7.5 \mathrm{GBd})$ then the quantization noise enhancement, and therefore the SNR penalty, is substantially lower when ZF is applied.

The obtained results indicate that the MMSE-based DPE can enable the targeted data rates of the udWDM-PON, up to 20 $\mathrm{Gb} / \mathrm{s}$, employing direct modulation of commercial DFBs with low-cost DACs.

\section{DSP SUBSYSTEMS OF THE RECEIVER}

The DSP inherited from the coherent RX for core networks in Fig. 2, can also be adapted and further simplified for the udWDM access scenario having different requirements and constraints, where the dominant transmission impairments mostly originate from the CoTRX photonic/electronic devices rather than the fiber channel.

In the coherent PON, hundreds of $\lambda$ s are efficiently allocated in ultra-dense grid configuration, achieving high aggregate PON capacity but retaining the data rate per single $\lambda$ down. Also, the use of spectrally-efficient modulation formats contributes to further reduce the signal $\mathrm{BW}$, thus minimizing the effect of $\mathrm{CD}$ and polarization mode dispersion (PMD), that have major impact when large signal BWs are transmitted over long fiber spans. Hence, CD and PMD are not yet the limiting factor for the udWDM access network, with fiber spans shorter than 40 or $60 \mathrm{~km} \mathrm{[1].}$

Owing to the superior sensitivity of coherent RXs, with -31.5 $\mathrm{dBm}$ as the worst case in Table $\mathrm{I}$, the launched power per $\lambda$ can be reduced below the threshold for NL impairments of the optical fiber [2], but still achieving the target loss budget $>30$ $\mathrm{dB}$ for a launched power of, e.g., $0 \mathrm{dBm}$ per user or even lower. 


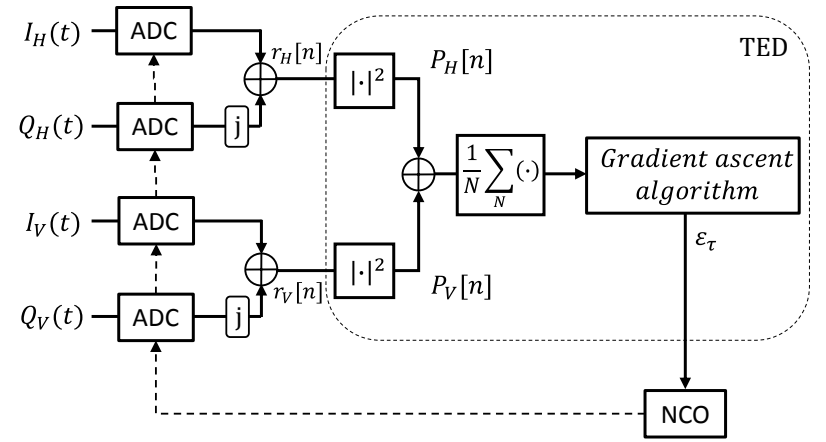

Fig. 9. Power-based CLK recovery to operate with symbol-rate ADCs.

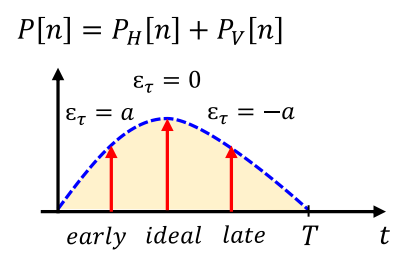

Fig. 10. Estimation of $\varepsilon_{\tau}$ from the calculated signal power to optimize the ADC sampling frequency.

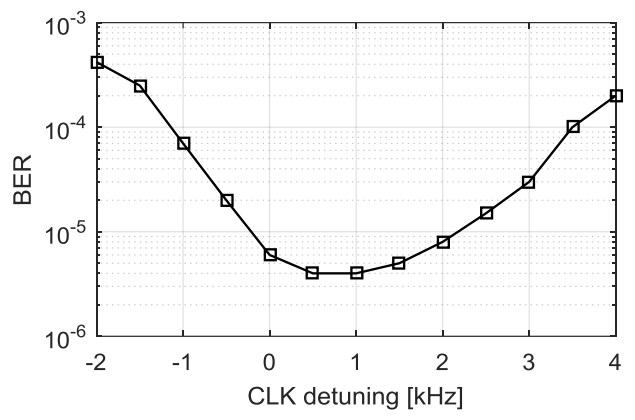

Fig. 11. BER vs. CLK detuning between TX and RX, for real-time BPSK data at $1.25 \mathrm{~Gb} / \mathrm{s}$ sampled by $1.25 \mathrm{GSa} / \mathrm{s}$ ADCs.

Last, front-end correction and orthonormalization for imbalanced optical hybrids are more critical for dense QAM constellations that are not feasible for PONs.

Therefore, the key DSP subsystems of the RX in Fig. 3 are: equalization for residual ISI, clock (CLK) recovery, and CR. In this section, equalization for residual ISI is not further discussed since it follows similar techniques than the DPE examined in Section III-C.

\section{A. Clock Recovery}

In the coherent RX, ADCs might not operate at the same speed as the received data. Therefore, CLK recovery is required to synchronize the received data with the DSP. Within the CLK recovery algorithm, the timing error detector (TED) estimates the optimal sampling phase that minimizes the timing error $\varepsilon_{\tau}$. A common non-data aided TED is the Gardner method, that requires $\times 2$ oversampling (i.e., two samples per symbol) to determine early, ideal and late sampling instants [27]. Hence, the sampling rate $R_{S}=2 B$, where $B$ is the $\mathrm{BW}$ of the photodetected signals. Fractional sampling $R_{S}=\gamma B$, with $1<$ $\gamma<2$, can also be performed to digitalize data, that are later resampled to $R_{S}=2 B$ by a digital interpolator at the DSP [28].

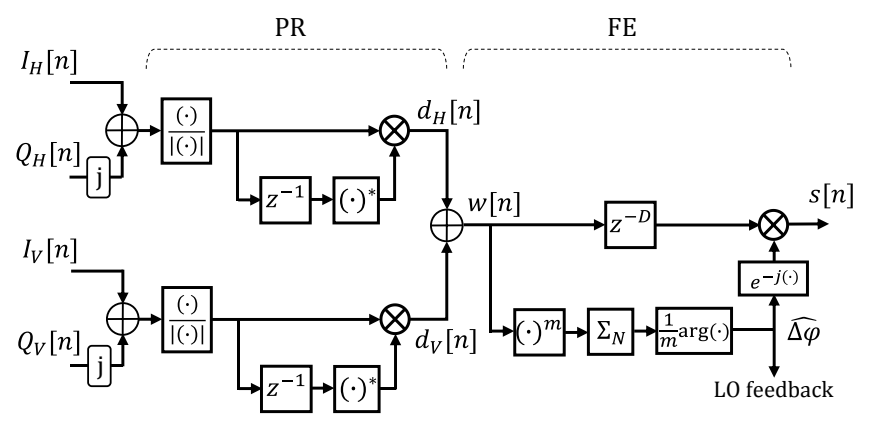

Fig. 12. Differential CR architecture for polarization-diversity $m$-PSK.

An alternative to Gardner was presented in [29], based on the power of the received signal. This power-based TED is particularly attractive in the access scenario because the estimation of the timing error is purely based on the signal power, then immune to the phase noise and frequency drifts from DFBs. To synchronize the CLK of the ADC with the received data, the TED works in a loop with a phase-locked loop (PLL) circuit or a numerically-controlled oscillator (NCO), to correctly tune the ADC sampling frequency.

Fig. 9 presents the block diagram of the CLK recovery algorithm that implements the power-based TED. First, the power of the received complex signals $r_{H}[n]$ and $r_{V}[n]$ for each SOP is calculated, then added to eliminate polarization effects. Next, averaging of the total signal power $P[n]=P_{H}[n]+$ $P_{V}[n]$ is carried out for noise suppression, followed by a gradient ascent algorithm that estimates $\varepsilon_{\tau}$ by maximizing $P[n]$. The estimated $\varepsilon_{\tau}$ drives a NCO which is fed-back to the ADC to dynamically adjust its sampling frequency. Therefore, in the CLK recovery architecture in Fig. 9 synchronization is achieved within the loop by looking at the ADC sampling frequency that maximizes $P[n]$, as represented in Fig. 10 .

The power-based CLK recovery was prototyped in a commercial FPGA for real-time evaluation. Direct BPSK modulation was generated from a pulse pattern generator (PPG) running at $1.25 \mathrm{~Gb} / \mathrm{s}$. Two DFBs with $\Delta v=4 \mathrm{MHz}$ each were used as TX and LO. At the RX, four ADC channels at 1.25 $\mathrm{GSa} / \mathrm{s}$ sampled the four photodetected signals. After photodetection, the signal $\mathrm{BW} B=R_{B}$ because, even for Nyquist filtering, the BW is not reduced to $R_{B} / 2$ due to the nonlinear phase modulation, as analyzed in Section III-B. Therefore, the ADCs operate at the symbol rate. In our specific implementation for FPGA, $\varepsilon_{\tau}$ drives a mixed-mode CLK manager (MMCM) that dynamically shifts the phase of a reference CLK embedded in the FPGA board, that drives the ADC sampling instants.

The CLK recovery tolerance against CLK detuning between TX and RX was evaluated in the real-time experiment with the FPGA. The received optical power was set to $-43 \mathrm{dBm}$, and the CLK frequency of the PPG was swept $\pm 4 \mathrm{kHz}$ with respect to $R_{B}$. The results in Fig. 11 show non-symmetric tolerance to CLK mismatch, with the lowest BER at $\sim 1 \mathrm{kHz}$ CLK detuning, mostly influenced by non-symmetric positive and negative slopes of the calculated $P[n]$ in Fig. 10, due to non-ideal HW frequency response. Moreover, the proposed CLK recovery 
algorithm operating at the symbol rate, tolerates up to $\pm 1 \mathrm{kHz}$ CLK detuning with respect to the optimal without significant BER degradation.

\section{B. Carrier Recovery}

In DSP for optical coherent RXs, there exist numerous algorithms to correctly recover the transmitted constellation in presence of optical phase noise and frequency drifts from the TX and LO lasers. The differential phase detection is a promising candidate due to its simplicity, robustness, high tolerance to the phase noise, and straightforward prototyping on the digital processor. If the phase noise is dominant at the decision part of the RX, e.g., when employing common DFBs or VCSELs with large $\Delta v$, the performance of differential phase detection is comparable to that of the synchronous detection [30], but with a remarkable lower implementation complexity. Moreover, although differential detection requires precoding at the TX, it represents no significant extra complexity and allows operating with free running LO without optical PLL.

Fig. 12 depicts the differential CR for the CoTRX in Fig. 3. It was proposed in [14] as a simplified CR architecture that merges in a single algorithm the differential demodulation for phase recovery (PR), and the differential $m$ th-power frequency estimation (FE), thus lowering the HW resources and process delay of the CR. For instance, when prototyping in a FPGA for DPSK data, the simplified CR requires $25 \%$ and $23 \%$ less real multipliers and adders respectively, and the CR process delay is reduced by $25 \%$, compared with the conventional CR with FE and PR unmerged, as reported in Table I of [14].

Within the CR algorithm in Fig. 12, digital samples of the received complex signal $I[n]+j Q[n]$ for each SOP are first normalized by its complex modulus in order to demodulate the phase information only, for the case of modulations formats with multiple radii. The amplitude information can be simultaneously recovered by an envelope detector. Next, the differential demodulation recovers the $m$-PSK data from the phase difference between consecutive symbols. Here, $m$ represents the order of the PSK modulation (e.g., $m=4$ for QPSK). This operation cancels the phase noise, as long as the symbol rate is several orders of magnitude larger than the total $\Delta v$ (TX + LO lasers), depending on the modulation format. Since the optical modulation at the TX in Fig. 3 is singlepolarization, the demodulated signals for each received SOP $d_{H}[n]$ and $d_{V}[n]$, are added to counteract variations of the transmitted SOP after propagating through the fiber.

After PR, each recovered symbol in $w[n]$ has a phase shift $\Delta \varphi=2 \pi \Delta f / R_{B}$ due to the frequency detuning between TX and LO lasers, denoted by $\Delta f$. It produces a fixed incremental rotation of the constellation in the complex plane, which is estimated and corrected by the FE block before symbol decision. The estimation of $\Delta \varphi$ is given by

$$
\widehat{\Delta \varphi}=\frac{1}{m} \arg \left[\frac{1}{N} \sum_{n=0}^{N-1}(w[n])^{m}\right]
$$

with $N$ the block length for averaging to counteract noise. In

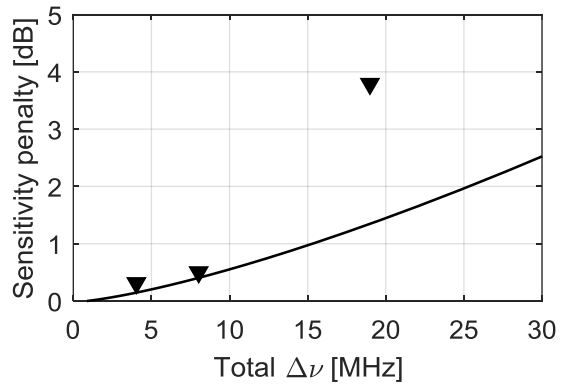

Fig. 13. Sensitivity penalty at BER $=10^{-3}$ vs. total $\Delta v$ for DPSK at $1.25 \mathrm{~Gb} / \mathrm{s}$; simulation (solid line) and real-time experimental results (marker).

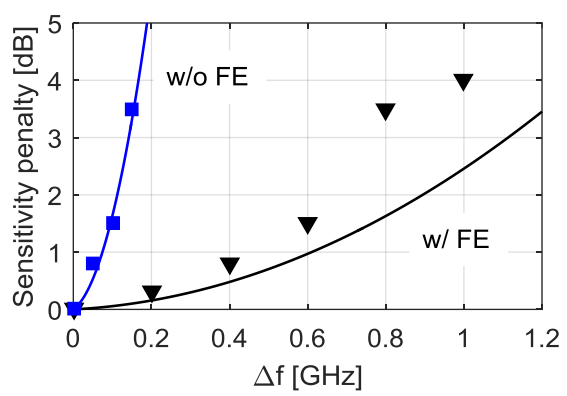

Fig. 14. Sensitivity penalty at $\mathrm{BER}=10^{-3}$ vs. frequency detuning $\Delta f$ for DPSK at $1.25 \mathrm{~Gb} / \mathrm{s}$, with and without $\mathrm{FE}$; simulation (solid line) and real-time experimental results (marker).

Fig. 12, $D$ stands for the process delay of the FE algorithm. The estimated $\widehat{\Delta \varphi}$ is able to drive both: the feed-forward symbol phase correction $w[n] \times \exp [-j \widehat{\Delta \varphi}]$, and the LO feed-back for automatic $\lambda$ tuning. After FE, the constellation is completely recovered within $s[n]$ and ready for the decision part of the RX.

The proposed CR algorithm is completely feed-forward; therefore, it is expected to be more robust against the phase noise and less challenging for parallel HW prototyping, compared with feed-back CR architectures. To validate the performance of the differential $\mathrm{CR}$ and its tolerance to phase noise and frequency drifts, it was implemented on FPGA for real-time evaluation with similar HW configuration than in previous Section IV-A. Differential BPSK (DPSK) data at 1.25 $\mathrm{Gb} / \mathrm{s}$ were generated by direct modulation of a DFB with $\Delta v=$ $4 \mathrm{MHz}$. Three different lasers with $\Delta v=\{0.1,4,15\} \mathrm{MHz}$ were used as LO, which translates into total $\Delta v=\{4,8,19\} \mathrm{MHz}$ respectively.

Note that $1.25 \mathrm{~Gb} / \mathrm{s}$, proposed for residential users in the udWDM-PON, is the most challenging scenario for the CR algorithm because $R_{B}$ is the closest to the total $\Delta v$, and therefore, the phase noise and the frequency drifts have stronger impact on the PSK data. In Fig. 13, the sensitivity penalty at $\mathrm{BER}=10^{-3}$ as a function of the total $\Delta v$ shows that the differential CR tolerates a ratio $\Delta v / R_{B}=0.64 \%$ for less than 1 $\mathrm{dB}$ sensitivity penalty, with $\Delta v=8 \mathrm{MHz}$ and DPSK data (experimental results). The obtained results are in the range of conventional low-cost DFBs, validating its feasibility for PON. Moreover, the achieved $\Delta v / R_{B}$ of the differential CR outperforms the real-time experiments reported in literature with synchronous detection, to the best of the author's 
knowledge. For higher order modulation formats at the same $R_{B}$, the $\Delta v$ tolerance is correspondingly lower. With off-line post-processing, the differential CR has also been demonstrated for up to 8-(A)PSK at $7.5 \mathrm{~Gb} / \mathrm{s}$, with total $\Delta v$ up to $2 \mathrm{MHz}$, achieving high RX sensitivity (see Table I).

Finally, the performance of the FE was evaluated in terms of the sensitivity penalty at $\mathrm{BER}=10^{-3}$ against $\Delta f$, with total $\Delta v$ $=8 \mathrm{MHz}$. As observed in Fig. 14, without FE only $\sim 50 \mathrm{MHz}$ detuning are tolerated for less than $1 \mathrm{~dB}$ penalty, but raises up to $\sim 400 \mathrm{MHz}$ after FE, necessary to correct the $\lambda$ drifts from DFBs due to temperature variations and laser aging. In all cases, experimental results are in reasonable agreement with numerical simulations, carried out under similar conditions.

\section{CONCLUSION}

We carried out a comprehensive analysis of the key enabling technologies to make the coherent systems affordable in complexity and cost, suitable for optical access networks and other applications with high terminal density, where simple CoTRXs are required. The novel class of CoTRX leverages consumer electronics and common DFBs with limited thermal tunability. The optical $m$-(A)PSK modulation is simplified by exploiting the photonic integration of DFB and EAM, enhancing the energy efficiency and lowering the footprint when compared to external IQ modulators.

The proposed CoTRX implements HW-efficient DSP, fundamental to overcome propagation impairments and increase the capacity and spectral efficiency of the PON. Within the DSP, we demonstrated simple pulse-shaping for direct phase modulation by the laser chirp, a DPE algorithm highly tolerant to quantization noise from band-limited and lowresolution DACs, a power-based CLK recovery that operates at the symbol rate, further lowering the ADC requirements, and a robust differential CR that tolerates up to $\Delta v=0.64 \%$ of the bit rate for DPSK in real-time.

Despite the simplified complexity of the CoTRX, the performance still retains high compared with the competing technologies for PON. The tested data rates span from 1.25 $\mathrm{Gb} / \mathrm{s}$ with BPSK to $7.5 \mathrm{~Gb} / \mathrm{s}$ with 8 -(A)PSK, in a $6.25 \mathrm{GHz}$ spaced udWDM optical grid for dedicated $\lambda$-to-the-user. The achieved Rx sensitivities for all the modulation formats yield a power loss budget $>30 \mathrm{~dB}$, when $0 \mathrm{dBm}$ launched power per user is considered. The digital transceiver potentially allows for fast reconfiguration enabling flexible optical grids for convergence of services, supporting residential and enterprises subscribers, as well as $\mathrm{MFH} / \mathrm{MBH}$ for $5 \mathrm{G}$ over the same PON infrastructure, fully compatible with legacy access systems.

\section{AKNOWLEDGMENT}

The authors would like to thank Victor Polo and Antonio Napoli for the fruitful discussions. S. Ghasemi would like to thank the Spanish FPI-Mineco, grant no. BES-2016-077129.

\section{REFERENCES}

[1] ITU-T G.989.2 Recommendation: "40-Gigabit-capable Passive Optical Networks (NG-PON2): Physical media dependent (PMD) layer specification", Feb. 2019.
[2] J. Prat et al, "Technologies for cost-effective udWDM-PONs," J. Lightw. Technol., vol. 34, no. 2, pp. 783-791, Jan. 15, 2016.

[3] M. Presi et al, "Field-trial of a high budget, filterless, $\lambda$-to-the-user, UDWDM-PON enabled by an innovative class of low-cost coherent transceivers," J. Lightw. Technol., vol. 35, no. 23, pp. 5250-5259, Dec. 1, 2017.

[4] V. Houtsma and D. van Veen, "Optical strategies for economical next generation 50 and 100G PON," in Proc. Conf. OFC, 2019, paper M2B.1.

[5] J. Cheng et al, "Comparison of coherent and IMDD transceivers for intra datacenter optical interconnects," in Proc. Conf. OFC, 2019, paper W1F.2.

[6] Optical Internetworking Forum (OIF), "Technology options for 400G implementation," Jul. 2015.

[7] M. Artiglia et al, "Coherent systems for low-cost $10 \mathrm{~Gb} / \mathrm{s}$ optical access networks," J. Lightw. Technol., vol. 33, no. 15, pp. 3338-3344, Aug. 2015.

[8] R. Ferreira et al, "Coherent UDWDM-PON with dual-polarization transceivers in real-time," J. Lightw. Technol., vol. 29, no. 11, pp. 909912, Jun. 1, 2017.

[9] W. Shi et al, "Silicon photonic modulators for high-capacity coherent transmissions," in Proc. Conf. OFC, 2019, paper Tu2H.1.

[10] M. S. Erkilinç et al, "Polarization-insensitive single-balanced photodiode coherent receiver for long-reach WDM-PONs," J. Lightw. Technol., vol. 34, no. 8, pp. 2034-2041, Apr. 15, 2016.

[11] K. Matsuda and N. Suzuki, "Hardware-efficient signal processing technologies for coherent PON systems," J. Lightw. Technol., vol. 37, no. 6, pp. 1614-1620, Mar. 15, 2019.

[12] A. Mecozzi, C. Antonelli, and M. Shtaif, "Kramers-Kronig coherent receiver," Optica, vol. 3, no. 11, pp. 1220-1227, Nov. 2016.

[13] J. C. Velásquez, J. Tabares, and J. Prat, "Differential 8-APSK monolithically integrated dual-EML transmitter for flexible coherent PONs," Opt. Letters, vol. 44, no. 11, pp. 2760-2763, Jun. 1, 2019.

[14] J. Tabares et al., "Simplified carrier recovery for intradyne optical PSK receivers in udWDM-PON," J. Lightw. Technol., vol. 36, no. 14, pp. 2941-2947, Jul. 15, 2018.

[15] K. Grobe et al., "Access networks based on tunable lasers," J. Lightw. Technol., vol. 32, no. 16, pp. 2815-2823, Aug. 15, 2014.

[16] V. Sales et al., "UDWDM-PON using low-cost coherent transceivers with limited tenability and heuristic DWA," IEEE J. Opt. Commun. Netw., vol. 8, no. 8, pp. 582-599, Aug. 2016.

[17] I. N. Cano, J. C. Velásquez, and J. Prat, "7.5 Gb/s direct DFB phase modulation with 8-DPSK for $6.25 \mathrm{GHz}$ spaced coherent UDWDMPONs," in Proc. Conf. OFC, 2016, pp. 1-3, paper M3C.4.

[18] J. C. Velásquez et al., "Simultaneous DPSK-ASK modulated dual-EML transmitter for coherent UDWDM-PON," in Proc. Conf. OFC, 2018, paper M3B.4.

[19] C. Henry, "Theory of the linewidth of semiconductor lasers," IEEE J. Quantum Electron, vol. QE-18, no. 2, pp. 259-264, Feb. 1982.

[20] R. Tucker, "High-speed modulation of semiconductor lasers," J. Lightw. Technol., vol. LT-3, no. 6, pp. 1180-1192, Dec. 1985.

[21] K. Petermann, Laser Diode Modulation and Noise, Dordrecht, the Netherlands, Kluwer Academic, 1988.

[22] J. C. Velásquez et al., "15-dB differential link-loss UDWDM-PON with direct beat phase modulated DFBs," IEEE Photon. Technol. Lett., vol. 30, no. 2, pp. 137-140, Jan. 15, 2018.

[23] J. C. Velásquez et al., "1.25-2.5 Gb/s simple Nyquist transmitters for coherent UDWDM-PON with enhanced spectral efficiency," Fiber and Integrated Optics, vol. 37, no. 4, pp. 219-228, Apr. 19, 2018.

[24] X. Chen et al., "Equalization of two-mode fiber based MIMO signals with larger receiver sets," Opt. Express, vol. 20, no. 26, pp. B413-B418, Dec. 10, 2012.

[25] J. Tabares et al., "Digital pre-emphasis for $10 \mathrm{~Gb} / \mathrm{s}$ with low-cost directly phase-modulated lasers for PONs," in Proc. Signal Process. Photon. Commun. (SPPCOM), 2018, paper SpTh3G.2.

[26] A. Napoli et al., "Digital compensation of bandwidth limitations for highspeed DACs and ADCs," J. Lightw. Technol., vol. 34, no. 13, pp. 30533064, Jul. 1, 2016.

[27] F. Gardner, "A BPSK/QPSK timing-error detector for sampled receivers," IEEE Trans. Commun., vol. COM-34, no. 5, pp. 423-429, May 1986.

[28] K. Kikuchi, "Fundamentals of coherent optical fiber communications," $J$. Lightw. Technol., vol. 34, no. 1, pp. 157-179, Jan. 1, 2016.

[29] M. Yan et al., "Digital clock recovery algorithm for Nyquist signal," in Proc. Conf. OFC/NFOEC, 2013, paper OTu2I.7.

[30] I. Fatadin, D. Ives, and S. Savory, "Differential carrier phase recovery for QPSK optical coherent systems with integrated tunable lasers," Opt. Exp., vol. 21, no. 8, pp. 10166-10171, 2013. 